\title{
Prognostic Value of Vitamin-D Level in Non-metastatic Breast Cancer Patients in Saudi Arabia
}

\author{
Shereef Elsamany ${ }^{1,2}$, Omaima Elemam ${ }^{1,2}$, Ahmed Zeeneldin ${ }^{1,3}$, Soha Elmorsy ${ }^{4,5}$ \\ Ahmed Khatry ${ }^{6}$, Faisal Alhuthali ${ }^{6}$, Suha Alsayed $^{6}$
}

${ }^{1}$ Oncology Center, King Abdullah Medical City, Makkah Saudi Arabia. ${ }^{2}$ Oncology Center, Mansoura University, Mansoura, Egypt. ${ }^{3}$ National Cancer Institute, Cairo University, Cairo, Egypt. ${ }^{4}$ Research Center, King Abdullah Medical City, Makkah Saudi Arabia. ${ }^{5}$ Pharmacology, Faculty of Medicine, Cairo University, Cairo, Egypt. ${ }^{6}$ Research, Um Alqura University, Makkah, Saudia Arabia.

\begin{abstract}
Background: Deficiency of vitamin-D (Vit-D) was associated with poor survival outcome in several studies across different tumour types. The present study aims to assess the prevalence and prognostic value of Vit-D deficiency among breast cancer patients in a single institution in Saudi Arabia. Methods: In this retrospective study, we screened patients who presented with non-metastatic breast cancer to King Abdullah Medical City, Saudi Arabia from June 2011 to December 2015. We checked baseline Vit-D level before starting systemic therapy in addition to other clinicopathological factors. Low Vit-D was defined as Vit-D level less than $30 \mathrm{ng} / \mathrm{ml}$. The relations of Vit-D level (taking the median as the cutoff) with clinicopathological factors were assessed using Chi-Square test. Differences in survival outcome were compared using log rank test. Results: We screened 340 patients with non-metastatic breast cancer. Baseline Vit-D levels were available for 189 patients. The median age was 50 years (range: $26-86$ years). Noteworthy, 169 (89.4\%) of patients had Vit-D level $<30 \mathrm{ng} / \mathrm{ml}$ with a median of $14.9 \mathrm{ng} / \mathrm{ml}$ (range: 4.0 - 45.0). Low Vit-D level (below the median) was significantly more common in premenopausal $(\mathrm{p}=0.011)$ and ER-negative patients $(\mathrm{p}=0.011)$. However, lymphovascular invasion $(\mathrm{p}=0.001)$, clinically $(\mathrm{p}=0.023)$ and pathologically positive axillary LNs $(\mathrm{p}=0.041)$ were linked with higher Vit- $\mathrm{D}$ level. After a median follow up period of 58.2 months, 14 patients died and 40 relapsed. The 5-year disease-free survival (DFS) rates was $74.8 \%$. The 5-year DFS rate in patients with higher Vit-D level above the median was $78.8 \%$ compared to $71.1 \%$ in patients with lower Vit-D level with no statistically significance difference $(p=0.22)$. The 5 -year overall survival (OS) rate was $90.2 \%$. Meanwhile, no difference in 5-year OS rate in patients with higher and lower Vit-D levels (90.3\% and 89.7\% respectively, $\mathrm{p}=0.6$ ). Conclusion: Low Vit-D level was prevalent among the studied breast cancer patients. Low Vit-D level was associated with ER-negative phenotype and premenopausal patients. Baseline Vit-D level was not significantly linked with survival outcome.
\end{abstract}

Keywords: Vitamin D- Breast Cancer- prognosis

Asian Pac J Cancer Care, 5 (1), 9-14

\section{Introduction}

Vitamin D (Vit-D) plays a vital role in calcium homeostasis, skeletal metabolism in addition to other vital physiological roles. Vit-D deficiency is a common health problem with numerous health consequences including osteomalacia, osteoporosis and fractures in adults [1].

Many reports showed an association between serum Vit-D deficiency and development of several types of cancer, including breast, colorectal, kidney and pancreatic
Submission Date: 11/05/2019 Acceptance Date: 01/04/2020

cancers [2-3]. Several studies have confirmed that vitamin D receptors (VDR) are expressed in normal breast tissues and also in breast cancer biopsy specimens [4-5]. Noteworthy, Vit-D promotes apoptosis through the insulin-like growth factor receptor 1 (IGFR)- (PI3K)Akt-dependent signaling pathway [3-6]. Therefore, deregulation of Vit-D signaling and related metabolic pathways was suggested to play an important role in tumour growth [7]. Meanwhile, large epidemiological studies suggested that Vit- D intake has a protective role

Corresponding Author:

Shereef Elsamany

Oncology Center, King Abdullah Medical City, Makkah Saudi Arabia.

Email: shereefmohamad@yahoo.com 
against breast cancer development [8-9].

However, the prognostic value of VDR expression and circulating Vit-D level still remains controversial. Several studies reported that deficiency of Vit-D was associated with poor survival outcome across different tumour types while other studies reported different conclusions [10-13]. The present study aims to assess the prognostic value of Vit-D deficiency among non-metastatic breast cancer patients in a single institution in Saudi Arabia. This may give rise to an easy prognostic parameter that can be assessed in daily practice.

\section{Materials and Methods}

\section{Study population}

Patients with histologically confirmed non-metastatic breast cancer who presented to King Abdullah Medical City, Saudi Arabia from June 2011 to December 2015, were included. Enrolled patients must have available baseline serum Vit-D level before starting any systemic therapy.

\section{Study design and procedures}

In this retrospective study, eligible patients must have adequate medical records. We checked baseline Vit-D level before starting systemic therapy in addition to other clinicopathological factors. Different parameters were collected including age, gender, stage at diagnosis, body mass index (BMI), pathological type, grade, ER, PR and HER2 status in addition to treatment data including type and number of chemotherapy cycles, hormonal therapy and trastuzumab (if applicable). Dates of disease relapse and death if any, were recorded.

\section{Statistical analysis}

SPSS version 21 statistical program was used. Descriptive statistics were performed for all clinical, laboratory and pathological data. Low Vit-D was defined as Vit-D level less than $30 \mathrm{ng} / \mathrm{ml}$. The relations of Vit-D level (taking $30 \mathrm{ng} / \mathrm{ml}$ and the median as the cutoffs) with clinico-pathological factors were assessed using Chi-Square test. Different potential prognostic factors were assessed in relation with disease free survival (DFS) and overall survival (OS). Survival data was presented by Kaplan Meier method where cases with no recorded events (death or relapse) were censored at the date of last contact. Comparisons of survival outcome among different parameters were assessed using the log rank test. A two-sided alpha was set at 0.05 . DFS was defined as the time from date of breast surgery to date of documented tumour relapse or death. OS was defined as the time from the date of diagnosis of breast cancer till the date of death.

\section{Results}

\section{Patients' and tumor characteristics}

We screened 340 patients with non-metastatic breast cancer. Baseline Vit-D levels were available for 189 patients with a median level of $14.9 \mathrm{ng} / \mathrm{ml}$ (range: 4.0 - 45.0). Noteworthy, $169(89.4 \%)$ of patients

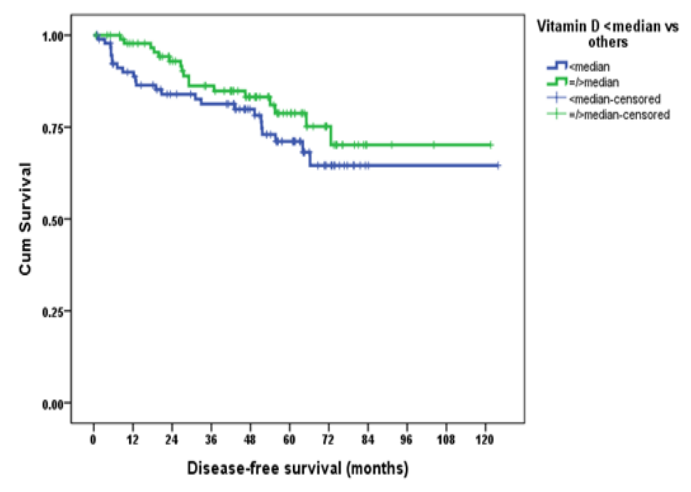

Figure 1. Disease-free Survival of Study Patients According to Vitamin D Level

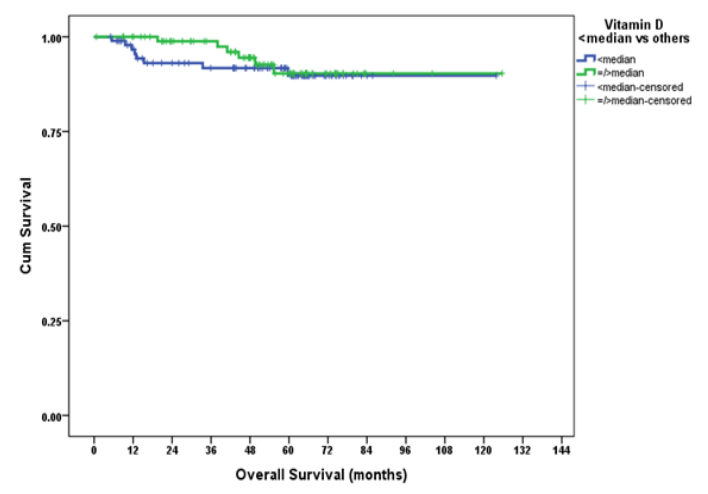

Figure 2. Overall Survival of Study Patients According to Vitamin D Level

had Vit-D level $<30 \mathrm{ng} / \mathrm{ml}$. Using $30 \mathrm{ng} / \mathrm{ml}$ as the cutoff, there was no significant association between different parameters and Vit-D level. We therefore, used the median Vit-D level as the cutoff to have enough patients for comparison. The median age was 50 years (range: 26- 86 years) and it was significantly lower in patients with lower compared to higher Vit-D levels (47 vs. 51 years respectively, $\mathrm{p}=0.04$ ). Similarly, Low Vit-D level (below the median) was significantly more common in premenopausal compared to postmenopausal patients (59.4\%, 40.9\%, respectively, $\mathrm{p}=0.01)$ and ER-negative vs. positive patients ( $63.1 \%$ vs $43.5 \%$, respectively, $\mathrm{p}=0.01)$. However, lymphovascular invasion (LVI) $(72.2 \%$ vs. $27.8 \%, \mathrm{p}=0.001)$, clinically $(62.5 \%$ vs. $37.5 \%$ respectively, $\mathrm{p}=0.02)$ and pathologically positive axillary LNs $(\mathrm{p}=0.04)$ were linked with higher Vit- D level. However, other clinicopathological factors did not significantly differ according to Vit-D level (Table 1).

\section{Survival outcome}

After a median follow up period of 58.2 months, 14 patients died and 40 relapsed. No difference in the rate of relapse between patients with lower vs. higher Vit-D level using different cutoffs, (Table 1). The 5-year DFS rate was $74.8 \%$. Using $30 \mathrm{ng} / \mathrm{ml}$ as the cutoff, no difference in DFS between higher and lower Vit-D levels (73.5\% vs. $75 \%, p=0.38$ ) was found. Using the median as the cutoff, 
Table 1. Baseline Patients' and Tumor Characteristics According to Vitamin D Level

\begin{tabular}{|c|c|c|c|c|}
\hline & $\begin{array}{c}\text { Total }=189 \\
\text { No }\end{array}$ & $\begin{array}{c}\text { Vitamin D } \\
< \\
\text { median } \\
\text { No (\%) }\end{array}$ & $\begin{array}{c}\text { Vitamin D } \\
\geq \\
\text { median } \\
\text { No (\%) }\end{array}$ & $\mathrm{P}$ \\
\hline \multicolumn{5}{|l|}{ Parameters } \\
\hline Median age (range) & $50(26-86)$ & $47(26-76)$ & $51(31-86)$ & 0.04 \\
\hline \multicolumn{5}{|l|}{ Menopausal status } \\
\hline Premenopausal & 96 & $57(59.4)$ & $39(40.6)$ & 0.01 \\
\hline Postmenopausal & 93 & $38(40.9)$ & $55(59.1)$ & \\
\hline \multicolumn{5}{|l|}{ Body mass index } \\
\hline$<25$ & 19 & $13(68.4)$ & $6(31.6)$ & \\
\hline $25-29.9$ & 69 & $31(44.9)$ & $38(55.1)$ & 0.25 \\
\hline $30-39.9$ & 79 & $38(48.1)$ & $41(51.9)$ & \\
\hline$\geq 40$ & 22 & $13(59.1)$ & $9(40.9)$ & \\
\hline \multicolumn{5}{|l|}{ Pathology } \\
\hline IDC & 178 & $89(50.0)$ & $89(50.0)$ & 0.07 \\
\hline ILC & 7 & $2(28.6)$ & $5(71.4)$ & \\
\hline Other & 4 & $4(100.0)$ & $0(0.0)$ & \\
\hline \multicolumn{5}{|l|}{ Grade } \\
\hline Grade 1 & 11 & $8(72.7)$ & $3(27.3)$ & \\
\hline Grade 2 & 104 & $51(49.0)$ & $53(51.0)$ & 0.07 \\
\hline Grade 3 & 63 & $34(54.0)$ & $29(46.0)$ & \\
\hline Unknown & 11 & $2(18.2)$ & $9(81.8)$ & \\
\hline \multicolumn{5}{|c|}{ Lymphovascular invasion } \\
\hline Yes & 54 & $15(27.8)$ & $39(72.2)$ & $<0.001$ \\
\hline No & 127 & $76(59.8)$ & $51(40.2)$ & \\
\hline \multicolumn{5}{|l|}{ ER status } \\
\hline Negative & 65 & $41(63.1)$ & $24(36.9)$ & \\
\hline Positive & 124 & $54(43.5)$ & $70(56.5)$ & 0.01 \\
\hline \multicolumn{5}{|l|}{ PR Status } \\
\hline Negative & 82 & $46(56.1)$ & $36(43.9)$ & 0.16 \\
\hline Positive & 107 & $49(45.8)$ & $58(54.2)$ & \\
\hline \multicolumn{5}{|l|}{ HER2 status } \\
\hline Negative & 124 & $62(50.0)$ & $62(50.0)$ & \\
\hline Positive & 62 & $33(53.2)$ & $29(46.8)$ & 0.19 \\
\hline Unknown & 3 & $0(0.0)$ & $3(100.0)$ & \\
\hline \multicolumn{5}{|l|}{ Clinical Stage } \\
\hline Stage I & 20 & $12(60.0)$ & $8(40.0)$ & \\
\hline Stage II & 88 & $41(46.6)$ & $47(53.4)$ & \\
\hline Stage IIIA & 52 & $30(57.7)$ & $22(42.3)$ & 0.42 \\
\hline Stage IIIB & 17 & $6(35.3)$ & $11(64.7)$ & \\
\hline Unknown & 12 & $6(50.0)$ & $6(50.0)$ & \\
\hline \multicolumn{5}{|l|}{ Clinical T stage } \\
\hline $\mathrm{Tx}$ & 13 & $8(61.5)$ & $5(38.5)$ & \\
\hline T0 & 9 & $4(44.4)$ & $5(55.6)$ & \\
\hline $\mathrm{T} 1$ & 32 & $18(56.3)$ & $14(43.8)$ & 0.18 \\
\hline $\mathrm{T} 2$ & 80 & $34(42.5)$ & $46(57.5)$ & \\
\hline $\mathrm{T} 3$ & 28 & $18(64.3)$ & $10(35.7)$ & \\
\hline $\mathrm{T} 4$ & 11 & $3(27.3)$ & $8(72.7)$ & \\
\hline \multicolumn{5}{|l|}{ Clinical LN status } \\
\hline Negative & 65 & $38(58.5)$ & $27(41.5)$ & \\
\hline Positive & 72 & $27(37.5)$ & $45(62.5)$ & 0.02 \\
\hline $\mathrm{Nx}$ & 52 & $30(57.7)$ & $22(42.3)$ & \\
\hline
\end{tabular}

Table 1. Continued

\begin{tabular}{|c|c|c|c|c|}
\hline & $\begin{array}{c}\text { Total }=189 \\
\text { No }\end{array}$ & $\begin{array}{c}\text { Vitamin D } \\
< \\
\text { median } \\
\text { No }(\%)\end{array}$ & $\begin{array}{c}\text { Vitamin D } \\
\geq \\
\text { median } \\
\text { No }(\%)\end{array}$ & $\mathrm{P}$ \\
\hline \multicolumn{5}{|c|}{ Pathological N } \\
\hline $\mathrm{Nx}$ & 5 & $1(20.0)$ & $4(80.0)$ & \\
\hline No & 91 & $54(59.3)$ & $37(40.7)$ & \\
\hline N1 & 49 & $18(36.7)$ & $31(63.3)$ & 0.04 \\
\hline $\mathrm{N} 2$ & 28 & $16(57.1)$ & $12(42.9)$ & \\
\hline $\mathrm{N} 3$ & 16 & $6(37.5)$ & $10(62.5)$ & \\
\hline \multicolumn{5}{|c|}{ Relapse } \\
\hline Yes & 40 & $23(57.5)$ & $17(42.5)$ & 0.30 \\
\hline No & 149 & $72(48.3)$ & $77(51.7)$ & \\
\hline
\end{tabular}

the 5 -year DFS rates were $78.8 \%$ vs. $71.1 \%$, in patients with higher compared to lower Vit-D levels, respectively. However, it did not reach statistical significance $(p=0.22)$ (Figure 1). Lower clinical $(\mathrm{p}=0.001)$ and pathological stages (stage I, II) (0.001) and higher BMI ( $\mathrm{p}=0.04)$ were the only factors associated with better DFS rates. Other clinico-pathological parameters were not linked with DFS outcome (Table 2).

The 5 -year OS rate was $90.2 \%$. Using $30 \mathrm{ng} / \mathrm{ml}$ as the cutoff, no difference in OS between higher and lower Vit-D levels $(93.5 \%$ vs. $85 \%, p=0.09)$ was found. Similarly, using the median as the cutoff, no difference in 5-year OS rate in patients with higher and lower Vit-D levels (90.3\% and $89.7 \%$ respectively, $p=0.6$ ) (Figure 2). OS was significantly higher in patients with lower pathologic stage (stage I, II), $(p=0.006)$. Meanwhile, no difference in OS outcome according to other clinico-pathological factors (Table 3).

\section{Discussion}

In this study, we assessed the prognostic value of baseline Vit-D level in a cohort of early breast cancer patients in a single institution in Saudi Arabia. Noteworthy, the great majority of patients $(89 \%)$ had low Vit-D level below the reference value and even half of the patients had considerably low values $(<14 \mathrm{ng} / \mathrm{ml})$. This highlights the prevalence of low Vit-D levels among Saudi patients with a median age of 50 years. This points to the magnitude of the problem of low Vit-D even among this cohort of generally young healthy patients in Saudi Arabia.

Noteworthy, lower Vit-D level was significantly linked with ER-negative phenotype and premenopausal status, features generally linked with more aggressive tumour behavior. Meanwhile, higher Vit-D values were linked with clinically and pathologically-positive lymph nodes and lymphovascular invasion. This conflicting data highlights the need to explore those findings in a larger cohort of patients.

Several studies reported an association between Vit-D deficiency and poor survival. In a prospective study including 512 patients with early breast cancer, low Vit-D was significantly linked with the risk of distant recurrence and overall survival [11]. Similarly, low Vit-D 
Table 2. Disease-free Survival in Various Subgroups

\begin{tabular}{|c|c|c|c|}
\hline Parameters & $\begin{array}{c}\text { Total }=189 \\
\text { No }\end{array}$ & $\begin{array}{c}\text { 5-year DFS } \\
(\%)\end{array}$ & $\mathrm{P}$ \\
\hline \multicolumn{4}{|l|}{ Body mass index } \\
\hline$<25$ & 19 & $81.7 \%$ & \multirow{4}{*}{0.04} \\
\hline $25-29.9$ & 69 & $63.2 \%$ & \\
\hline $30-39.9$ & 79 & $82.6 \%$ & \\
\hline$\geq 40$ & 22 & $79.5 \%$ & \\
\hline \multicolumn{4}{|l|}{ Menopausal status } \\
\hline Premenopausal & 96 & $70.4 \%$ & \multirow[t]{2}{*}{0.27} \\
\hline Postmenopausal & 93 & $78.9 \%$ & \\
\hline \multicolumn{4}{|l|}{ Clinical Stage } \\
\hline Stag I-II & 108 & $83.0 \%$ & \multirow[t]{2}{*}{0.001} \\
\hline Stage III & 69 & $66.1 \%$ & \\
\hline \multicolumn{4}{|l|}{ Clinical T } \\
\hline T0-2 & 121 & $80.6 \%$ & \multirow[t]{2}{*}{$<0.001$} \\
\hline T3-4 & 39 & $58.9 \%$ & \\
\hline \multicolumn{4}{|l|}{ Clinical LN status } \\
\hline Negative & 65 & $77.1 \%$ & \multirow{3}{*}{0.70} \\
\hline Positive & 72 & $74.2 \%$ & \\
\hline $\mathrm{Nx}$ & 52 & $74.0 \%$ & \\
\hline \multicolumn{4}{|l|}{ Pathology } \\
\hline IDC & 178 & $74.6 \%$ & \multirow{3}{*}{0.42} \\
\hline ILC & 7 & $60.0 \%$ & \\
\hline Other & 4 & $100.0 \%$ & \\
\hline \multicolumn{4}{|l|}{ Multicentricity } \\
\hline Yes & 26 & $61.7 \%$ & \multirow[t]{2}{*}{0.22} \\
\hline No & 163 & $76.5 \%$ & \\
\hline \multicolumn{4}{|c|}{ Lymphovascular invasion } \\
\hline Yes & 54 & $69.1 \%$ & \multirow[b]{2}{*}{0.11} \\
\hline No & 127 & $77.1 \%$ & \\
\hline \multicolumn{4}{|l|}{ Grade } \\
\hline Grade 1 & 11 & $66.7 \%$ & \multirow{4}{*}{0.51} \\
\hline Grade 2 & 104 & $74.2 \%$ & \\
\hline Grade 3 & 63 & $80.8 \%$ & \\
\hline Unknown & 11 & $62.3 \%$ & \\
\hline \multicolumn{4}{|l|}{ ER status } \\
\hline Negative & 65 & $70.8 \%$ & \multirow[t]{2}{*}{0.18} \\
\hline Positive & 124 & $76.7 \%$ & \\
\hline \multicolumn{4}{|l|}{ PR Status } \\
\hline Negative & 82 & $72.3 \%$ & \multirow[t]{2}{*}{0.40} \\
\hline Positive & 107 & $76.5 \%$ & \\
\hline HER2 status & & & \\
\hline Negative & 124 & $77.9 \%$ & \\
\hline Positive & 62 & $70.1 \%$ & 0.28 \\
\hline Unknown & 3 & $00.0 \%$ & \\
\hline Pathologic T stage & & & \\
\hline T0-2 & 159 & $76.2 \%$ & 0.04 \\
\hline T3-4 & 24 & $65.2 \%$ & \\
\hline Pathological N & & & \\
\hline N0 & 91 & $86.8 \%$ & 0.001 \\
\hline $\mathrm{N}+$ & 93 & $62.3 \%$ & \\
\hline
\end{tabular}

Table 2. Continued

\begin{tabular}{lccc}
\hline Parameters & $\begin{array}{c}\text { Total=189 } \\
\text { No }\end{array}$ & $\begin{array}{c}5 \text {-year DFS } \\
\text { (\%) }\end{array}$ & P \\
\hline Pathological Stage & & & \\
$\quad$ Stage 0-II & 130 & $79.6 \%$ & 0.02 \\
$\quad$ Stage III & 57 & $65.2 \%$ & \\
Chemotherapy cycles Number & & & \\
$\quad \leq 6$ & 74 & $70.6 \%$ & 0.67 \\
$\quad>6$ & 91 & 74.3 & \\
Chemotherapy Type & & & \\
$\quad$ Anthracycline \& Taxane & 135 & $70.9 \%$ & \\
$\quad$ Anthracycline only & 23 & $78.3 \%$ & 0.69 \\
$\quad$ Taxane only & 7 & $83.3 \%$ & \\
Vitamin D & & & \\
$\quad<$ median & 95 & $71.1 \%$ & 0.22 \\
$\quad \geq$ median & 94 & $78.8 \%$ & \\
\hline & & &
\end{tabular}

Table 3. Overall Survival (OS) in Various Subgroups

\begin{tabular}{|c|c|c|c|}
\hline Parameters & $\begin{array}{c}\text { Total } \\
189\end{array}$ & $\begin{array}{c}5 \text {-year OS } \\
(\%)\end{array}$ & $\mathrm{P}$ \\
\hline \multicolumn{4}{|l|}{ Body mass index } \\
\hline$<25$ & 19 & $92.9 \%$ & \multirow{4}{*}{0.17} \\
\hline 25-29.9 & 69 & $85.3 \%$ & \\
\hline 30-39.9 & 79 & $91.2 \%$ & \\
\hline$\geq 40$ & 22 & $100.0 \%$ & \\
\hline \multicolumn{4}{|l|}{ Body mass index } \\
\hline$<30$ & 88 & $86.7 \%$ & \multirow[t]{2}{*}{0.06} \\
\hline$\geq 30$ & 101 & $93.0 \%$ & \\
\hline \multicolumn{4}{|l|}{ Clinical Stage } \\
\hline Stag I-II & 108 & $92.0 \%$ & \multirow[t]{2}{*}{0.16} \\
\hline Stage III & 69 & $87.2 \%$ & \\
\hline \multicolumn{4}{|l|}{ Pathology } \\
\hline IDC & 178 & $91.1 \%$ & \multirow{3}{*}{0.05} \\
\hline ILC & 7 & $53.3 \%$ & \\
\hline Other & 4 & $100.0 \%$ & \\
\hline \multicolumn{4}{|l|}{ Menopausal status } \\
\hline Premenopausal & 96 & $86.6 \%$ & \multirow[t]{2}{*}{0.18} \\
\hline Postmenopausal & 93 & $93.4 \%$ & \\
\hline \multicolumn{4}{|l|}{ Clinical T } \\
\hline T0-2 & 121 & $92.8 \%$ & \multirow[t]{2}{*}{0.17} \\
\hline T3-4 & 39 & $87.3 \%$ & \\
\hline \multicolumn{4}{|c|}{ Lymphovascular invasion } \\
\hline Yes & 54 & $85.9 \%$ & \multirow[t]{2}{*}{0.13} \\
\hline No & 127 & $92.2 \%$ & \\
\hline \multicolumn{4}{|l|}{ Grade } \\
\hline Grade 1 & 11 & $100.0 \%$ & \multirow{4}{*}{0.52} \\
\hline Grade 2 & 104 & $87.0 \%$ & \\
\hline Grade 3 & 63 & $94.9 \%$ & \\
\hline Unknown & 11 & $87.5 \%$ & \\
\hline \multicolumn{4}{|l|}{ Clinical LN status } \\
\hline Negative & 65 & $91.3 \%$ & \multirow{3}{*}{0.79} \\
\hline Positive & 72 & $89.0 \%$ & \\
\hline $\mathrm{Nx}$ & 52 & $91.0 \%$ & \\
\hline
\end{tabular}


Table 3. Continued

\begin{tabular}{|c|c|c|c|}
\hline Parameters & $\begin{array}{c}\text { Total }=189 \\
\text { No }\end{array}$ & $\begin{array}{c}\text { 5-year DFS } \\
(\%)\end{array}$ & $\mathrm{P}$ \\
\hline \multicolumn{4}{|l|}{ ER status } \\
\hline Negative & 65 & $86.8 \%$ & 0.13 \\
\hline Positive & 124 & $91.8 \%$ & \\
\hline \multicolumn{4}{|l|}{ PR Status } \\
\hline Negative & 82 & $87.6 \%$ & 0.21 \\
\hline Positive & 107 & $91.8 \%$ & \\
\hline \multicolumn{4}{|l|}{ HER2 status } \\
\hline Negative & 124 & $93.3 \%$ & \\
\hline Positive & 62 & $85.9 \%$ & 0.14 \\
\hline Unknown & 3 & $0.00 \%$ & \\
\hline \multicolumn{4}{|l|}{ Pathological T } \\
\hline $\mathrm{T} 0-2$ & 159 & $91.1 \%$ & 0.22 \\
\hline $\mathrm{T} 3-4$ & 24 & $85.7 \%$ & \\
\hline \multicolumn{4}{|l|}{ Pathological N } \\
\hline No & 91 & $94.0 \%$ & 0.09 \\
\hline $\mathrm{N}+$ & 93 & $87.5 \%$ & \\
\hline \multicolumn{4}{|l|}{ Pathologic Stage } \\
\hline Stage 0-II & 130 & $94.0 \%$ & 0.01 \\
\hline Stage III & 57 & $82.9 \%$ & \\
\hline \multicolumn{4}{|l|}{ Chemotherapy } \\
\hline Yes & 165 & $90.4 \%$ & 0.95 \\
\hline No & 24 & $86.3 \%$ & \\
\hline \multicolumn{4}{|l|}{ Chemotherapy cycles Number } \\
\hline$\leq 6$ & 74 & $90.4 \%$ & 0.87 \\
\hline$>6$ & 91 & $90.5 \%$ & \\
\hline \multicolumn{4}{|l|}{ Chemotherapy Type } \\
\hline Anthracycline \& Taxane & 135 & $89.0 \%$ & \\
\hline Anthracycline only & 23 & $95.0 \%$ & 0.61 \\
\hline Taxane only & 7 & $100.0 \%$ & \\
\hline \multicolumn{4}{|l|}{ Vitamin D } \\
\hline$<$ median & 95 & $89.7 \%$ & 0.60 \\
\hline$\geq$ median & 94 & $90.3 \%$ & \\
\hline
\end{tabular}

level was significantly associated with poor OS and DFS in a larger cohort of 1,295 postmenopausal breast cancer patients [13]. However, despite the fact that several epidemiologic and clinical studies suggested that Vit- D deficiency may be associated with breast cancer outcome, other studies did not display any association. This may be attributed to timing of measurement of the vitamin D, stage, menopausal status and hormonal receptor status [14]. Noteworthy, the association of Vit-D level and survival outcome were assesses in two meta-analyses, involving 8 and 5 studies. These meta-analyses showed an association of low Vit-D level with recurrence in addition to overall and breast cancer-specific mortality in breast cancer patients [15-16].

In our study, Vit-D level was not linked with survival outcome. Meanwhile, almost all patients with low Vit-D, received calcium and Vit-D supplementation later on in their disease course, which may mitigate or modulate any potential prognostic value. Furthermore, only 10\% of patients had normal Vit-D levels above the reference value which limits the validity of comparing the survival outcome of low vs. normal Vit-D levels in our study. Meanwhile, there was a trend towards improved DFS in those with higher Vit-D level taking the median as the cutoff. However, that analysis was actually comparing low vs. higher (but still almost lower than normal value). Furthermore, only 14 patients died among the study population, which limits assessment of OS as data is still immature for OS comparison.

In conclusion, low Vit-D level was prevalent among the studied breast cancer patients. Low Vit-D level was associated with ER-negative phenotype and premenopausal patients. Baseline Vit-D level was not significantly linked with survival outcome.

\section{Acknowledgements}

-This research did not receive any specific grant from funding agencies in the public, commercial, or not-forprofit sectors.

-The authors declare no conflict of interest.

\section{References}

1. Atoum M and Alzoughool F. Vitamin D and Breast Cancer: Latest Evidence and Future Steps. Breast Cancer: Basic and Clinical Research 2017, 11: 1-8.

2. Wu X, Zhou T, Cao N, Ni J, Wang X. Role of vitamin D metabolism and activity on carcinogenesis. Oncol Res 2014;22:129-37.

3. Deeb KK, Trump DL, Johnson CS. Vitamin D signalling pathways in cancer: potential for anticancer therapeutics. Nat Rev Cancer 2007;7:684-700.

4. Freake HC, Abeyasekera G, Iwasaki J, Marcocci C, MacIntyre I, et al. Measurement of 1,25-dihydroxyvitamin D3 receptors in breast cancer and their relationship to biochemical and clinical indices.Cancer Res 1984;44:1677-81.

5. Lopes N, Paredes J, Costa JL, Ylstra B, Schmitt F. Vitamin $\mathrm{D}$ and the mammary gland: a review on its role in normal development and breast cancer. Breast Cancer Res 2012;14:211-4.

6. Ameri P, Giusti A, Boschetti M, Murialdo G, Minuto F, et al. Interactions between vitamin $\mathrm{D}$ and IGF-I: from physiology to clinical practice. Clin Endocrinol 2013;79:457-63.

7. Buttigliero C, Monagheddu C, Petroni P, Saini A, Dogliotti L et al. Prognostic role of vitamin D status and effcacy of vitamin D supplementation in cancer patients: a systematic review. Oncologist 2011;16:1215-27.

8. Peppone LJ, Rickles AS, Janelsins MC, Insalaco MR, Skinner KA. The association between breast cancer prognostic indicators and serum 25-OH vitamin D levels. Ann Surg Oncol2012;19:2590-9.

9. Rainville C, Khan Y, Tisman G. Triple negative breast cancer patients presenting with low serum vitamin D levels: a case series. Cases J 2009;2:8390-4.

10. Soljic M, Mrklic I, Tomic S, Omrcen T, Sutalo N, et al. Prognostic value of vitamin D receptor and insulinlike growth factor receptor 1 expression in triple negative breast cancer. J Clin Patho 2017, 10:1-6.

11. Goodwin PJ, Ennis M, Pritchard KI, Koo J, Hood N. Prognostic effects of 25-hydroxyvitamin D levels in early breast cancer. J Clin Oncol Off J Am Soc Clin Oncol 2009, 


\section{7, 3757-63.}

12. Kim HJ, Lee YM, Ko BS, Lee JW, Yu JH, et al. Vitamin $\mathrm{D}$ defciency is correlated with poor outcomes in patients with luminal-type breast cancer. Ann SurgOncol 2011, 18, $1830-6$.

13. Vrieling A, Hein R, Abbas S, Schneeweiss A, Flesch-Janys $\mathrm{D}$, et al. Serum 25-hydroxyvitamin D and postmenopausal breast cancer survival: a prospective patient cohort study. Breast Cancer Res BCR 2011, 13, R74.

14. Ismail1 A, El-Awady R, Mohamed G, Hussein M, Ramadan S. Prognostic Signifcance of Serum Vitamin D Levels in Egyptian Females with Breast Cancer Asian Pac J Cancer Prev, 19 (2), 571-576.

15. Vrieling A, Seibold P, Johnson TS, Heinz J, Obi N, et al. Circulating 25-hydroxyvitamin D and postmenopausal breast cancer survival: Influence of tumor characteristics and lifestyle factors?. Int J Cancer 2014, 134, 2972-83.

16. Maalmi H, Ordóñez-Mena JM, Schöttker B, Brenner H. Serum 25-hydroxyvitamin D levels and survival in colorectal and breast cancer patients: systematic review and metaanalysis of prospective cohort studies. Eur J Cancer Oxf Engl 2014, 50, 1510-21.

\section{(i) (3)}

This work is licensed under a Creative Commons AttributionNon Commercial 4.0 International License. 